\title{
Early readmission and length of hospitalization practices in the Dialysis Outcomes and Practice Patterns Study (DOPPS)
}

\author{
Antonio Alberto LOPES, ${ }^{1,2}$ Sean F LEAVEY, ${ }^{3}$ Keith McCULLOUGH, ${ }^{4}$ Brenda GILLESPIE, ${ }^{5}$ \\ Juergen BOMMER, ${ }^{6}$ Bernard J CANAUD, ${ }^{7}$ Akira SAITO, ${ }^{8}$ Shunichi FUKUHARA, ${ }^{9}$ \\ Philip J HELD, ${ }^{4}$ Friedrich K PORT, ${ }^{4}$ Eric W YOUNG ${ }^{10}$ \\ ${ }^{1}$ Department of Medicine, Federal University of Bahia, Brazil, and Visiting Professor, University of Michigan, \\ Ann Arbor, Michigan, U.S.A.; ${ }^{2}$ University of Michigan Kidney Epidemiology and Cost Center (KECC), Ann \\ Arbor, Michigan, U.S.A.; ${ }^{3}$ Waterford Regional Hospital, Ireland; ${ }^{4}$ University Renal Research and Education \\ Association (URREA), Ann Arbor, Michigan, U.S.A.; ${ }^{5}$ Department of Biostatistics, School of Public Health, \\ University of Michigan, Ann Arbor, Michigan, U.S.A.; ${ }^{6}$ University of Heidelberg, Heidelberg, Germany; \\ ${ }^{7}$ Lapeyronie University Hospital, Montpellier, France; ${ }^{8}$ Tokai University School of Medicine, Kanagawa, \\ Japan; ${ }^{9}$ Kyoto University, Kyoto, Japan; ${ }^{10}$ Division of Nephrology, VA Medical Center and University of \\ Michigan, Ann Arbor, Michigan, U.S.A.
}

\begin{abstract}
Background: Rising hospital care costs have created pressure to shorten hospital stays and emphasize outpatient care. This study tests the hypothesis that shorter median length of stay (LOS) as a dialysis facility practice is associated with higher rates of early readmission.

Methods: Readmission within 30 days of each hospitalization was evaluated for participants in the Dialysis Outcomes and Practice Patterns Study, an observational study of randomly selected hemodialysis patients in the United States (142 facilities, 5095 patients with hospitalizations), five European countries (101 facilities, 2281 patients with hospitalizations), and Japan (58 facilities, 883 patients with hospitalizations). Associations between median facility LOS (estimated from all hospitalizations at the facility and interpreted as a dialysis facility practice pattern) and odds of readmission were assessed using logistic regression, adjusted for patient characteristics and the LOS of each index hospitalization. Results: Risk of readmission was directly and significantly associated with LOS of the index hospitalization (adjusted odds ratio [AOR] 1.005 per day in median facility LOS, $p=0.007$ ) and inversely associated with median facility $L O S(A O R=0.974$ per day, $p=0.016)$. This latter association was strongest for US hemodialysis centers ( $A O R=0.954$ per day, $p=0.015$ ).

Conclusions: Dialysis facilities with shorter median hospital LOS for their patients have higher odds of readmission, particularly in the United States, where there is greater pressure to shorten LOS. The determinants and consequences of practices related to hospital LOS for hemodialysis patients should be further studied.
\end{abstract}

Key words: End-stage renal disease, readmission

Correspondence to: Eric W. Young, MD, MS, Department of Veterans Affairs Medical Center, Office of the Chief of Staff (11), 2215 Fuller Road, Ann Arbor, MI 48105, U.S.A.

E-mail: eyoung@umich.edu

\section{INTRODUCTION}

Hemodialysis patients face an increased risk of hospitalization because of a high prevalence of comorbid 
conditions and treatment-related complications. ${ }^{1-5}$ National data for end-stage renal disease patients treated in the United States show a decrease in the number of days per admission from 1993 to 1997, after adjusting for age, sex, race, and the diagnosis of diabetes mellitus. ${ }^{6}$ By contrast, the average annual hospitalization cost per patient has increased over time. The trend toward shorter hospital length of stay (LOS) for dialysis patients is consistent with incentives to reduce health care costs. ${ }^{6}$

The pressure to shorten hospitalization stays may lead to premature discharge in some cases. ${ }^{7-9}$ A potential consequence of premature discharge is an increase in unplanned early readmissions, a proposed marker of the quality of in-hospital and postdischarge care. ${ }^{10,11}$ The consequences of premature discharge may be worse for dialysis patients (compared to several other groups of patients) because of their high prevalence of comorbid conditions and increased risk of complications. ${ }^{12-18}$

Hemodialysis facilities provide a unique opportunity for evaluating the effect of hospital LOS practice on readmission risk because they function as logically unified practice units. Hospitalization practices for patients receiving regular treatment in a dialysis facility typically involve a small and cohesive group of providers, consultants, and hospitals. Furthermore, the hospital LOS practice of a dialysis facility can be measured with reasonable accuracy because dialysis patients as a group require frequent hospitalization. ${ }^{4}$ The facility LOS practice pattern is captured by the median LOS of all hospitalizations of facility patients. Practitioners associated with dialysis facilities with a high median LOS tend to keep their patients in the hospital longer than do practitioners working in facilities with a shorter median LOS. The concept of LOS as a practice pattern seems to be especially appropriate for hemodialysis patients because their care is largely organized at the dialysis facility level and they are hospitalized more frequently than many other patient groups. Hospital LOS is more difficult to express as a practice pattern for other patient groups because the practice unit is typically a single physician, and hospitalization is relatively infrequent.

This study examines the relationship between readmission and LOS for hemodialysis patients, using nationally representative samples of dialysis facilities and patients from France, Germany, Italy, Japan, Spain, the United Kingdom, and the United States. The LOS for each individual hospitalization was interpreted as an indicator of the severity of the condition for which each patient was hospitalized. The median LOS for each dialysis facility was interpreted as a marker of the local practice pattern. The study hypothesized that the risk of readmission was directly associated with individual hospital LOS (severity of disease) and inversely associated with median facility LOS (practice pattern).

\section{MATERIALS AND METHODS}

The data used for the present analysis were from the Dialysis Outcomes and Practice Patterns Study (DOPPS I), an international, prospective, observational study of hemodialysis practice patterns and associated outcomes. The study is ongoing in the United States (US-DOPPS), five European countries (referred to collectively as EuroDOPPS: France, Germany, Italy, Spain, and the United Kingdom), and Japan (Japan-DOPPS). ${ }^{19}$ Nationally representative samples of dialysis facilities were recruited in each country. The study was reviewed and approved by the appropriate institutional review boards in each country and in selected facilities. The overall design of the DOPPS has been described previously. ${ }^{19}$

The current study included data from 142 hemodialysis facilities in the United States (5095 patients with at least one hospitalization), 101 facilities in Europe (2281 patients with hospitalizations), and 58 facilities in Japan (883 patients with hospitalizations). Data collection began in 1997 in the United States, 1998 in Europe, and 1999 in Japan. Patients were replaced on an ongoing basis as they left participating facilities for reasons of death, transplantation, change in treatment modality, withdrawal from dialysis, recovery of renal function, or transfer to another facility.

At the time of enrollment of each new patient, the medical record was abstracted for extensive information about demographic characteristics, comorbid conditions, laboratory values, and renal diagnosis. Follow-up information was obtained approximately every 4 months including dates, diagnoses, and procedures associated with each hospitalization.

For hospitalizations during the study period with at least 30 days of postdischarge follow-up, a readmission was defined as a hospital admission within 30 days of the previous discharge. Readmission risk was modeled using logistic regression. Potential associations among the probabilities of readmission for multiple hospitalizations of the same patient were modeled using a compound symmetry (i.e., exchangeable) correlation structure. SAS software, version 8 (SAS Institute; Cary, NC), was used for all analyses.

The facility practice pattern for hospital LOS was estimated as the median LOS of all hospitalizations of patients at that facility, excluding hospitalizations associated with death. The logistic models of readmission risk 
simultaneously included both the LOS associated with each hospitalization (hospital LOS) and the dialysis facility median LOS (as a facility practice pattern). Models were adjusted for 27 covariates, including demographic characteristics, socioeconomic factors, reason for hospitalization, comorbid conditions, dialysis dose (estimated as the equilibrated $\left.\mathrm{Kt} / \mathrm{V}_{\text {urea }}\left[\mathrm{eKt} / \mathrm{V}_{\text {urea }}\right]\right)^{20}$ hematocrit, phosphorus, years on dialysis, and the country where the patient was treated. Hospital and facility median LOS were modeled as both categorical and continuous variables to fully explore the functional relationships of interest. The primary reason for each hospital admission was grouped (on the data collection code sheet) into the following 15 categories: infectious disease, vascular access, coronary heart disease, other cardiac disease (i.e., congestive heart failure, arrhythmias, and valvular and pericardial disease), peripheral vascular disease, cerebrovascular or neurologic disease, musculoskeletal disease, pulmonary disease, gastrointestinal or hepatic disease, endocrine or metabolic disease, cancer, visual disease, trauma or injury-related condition, psychiatric disease, and health maintenance or routine care.

\section{RESULTS}

Table 1 shows the baseline patient characteristics that were used for model-adjustment purposes. In general, the patient sample was enriched with elderly patients with multiple comorbid conditions, representative of the chronic hemodialysis population. The mean patient age was $61.5 \pm 14.8$ (SD) years. The sample composition was $21.5 \%$ black and $56.0 \%$ male. More than $50 \%$ of the patients were on dialysis for less than 1 year. Almost one-fourth of patients were living alone or in a setting indicative of diminished independence or social support. The prevalence of comorbid conditions was high, particularly for hypertension, coronary artery disease, diabetes, and congestive heart failure. As expected, large variation was seen in patient measurements, such as body mass index, dialysis dose, serum phosphorus and albumin concentration, and the blood hematocrit. These variables were included in the readmission models because they are known predictors of patient morbidity.

The overall hospital readmission rate was $26.2 \%$ (Table 2). Of these readmissions, $44.3 \%$ were grouped in the same diagnostic category as the initial hospitalization. The percentage of patients who were readmitted varied by diagnostic category of the initial hospitalization, ranging from $12.2 \%$ for vision-related hospitalizations to $33.1 \%$ for hospitalizations related to cancer. Readmis- sions within 30 days were frequently categorized in the same diagnostic group as the initial hospitalization, particularly for hospitalizations related to cancer (66.4\%), vascular access (56.7\%), and peripheral vascular disease (50.4\%). Because reasons for hospitalization were not independent, reasons for initial hospital admission or readmission that were considered likely to be related were combined. Data (not presented in Table 2) showed that $63.4 \%$ of patients with an initial admission caused by coronary heart disease or other cardiac diseases were readmitted within 30 days for one of these same initial reasons. These two cardiac causes were also combined with peripheral vascular disease and cerebrovascular/ neurologic disease; $72.4 \%$ of patients with one of these reasons for the index hospitalization had a readmission within 30 days attributable to one of these problems. Information about the reason for the initial admission was missing for $10 \%$ of hospitalizations.

The percentages of hospitalizations with LOS less than 3,3 to 4,5 to 9 , and more than 9 days were $29 \%$, $20 \%, 25 \%$, and $26 \%$, respectively. Hospital LOS also was characterized as a facility practice pattern, expressed as the median LOS for hospitalizations of facility patients that did not result in death. The median facility LOS estimated the overall hospitalization duration practice for a facility. Table 3 shows that the median facility LOS was shortest for US-DOPPS centers (median 4 days) and longest for Japan-DOPPS (median 12.5 days). Median LOS equal to or shorter than 5 days was seen in $85 \%$ of facilities in US-DOPPS, $48 \%$ in Euro-DOPPS, and 5\% in Japan-DOPPS.

Figure 1 displays the association between readmission risk and LOS, adjusted for patient characteristics (shown in Table 1), reason for hospitalization (Table 2), and country. The risk of readmission (expressed as the adjusted odds ratio [AOR]) was directly associated with the length of the initial (index) hospitalization (Fig. 1A). The readmission risk increased with index hospitalization LOS in a dose-dependent fashion for LOS greater than 4 days; admissions longer than 9 days had significantly greater risk of readmission than did admissions shorter than 3 days (AOR 1.18; $p=0.001$ ). In contrast, Fig. 1B shows that, when the data of the whole group of patients in the facility were used to determine the median LOS, facilities with longer LOS had lower odds of readmission. The odds of readmission were significantly lower (12\%) for dialysis facilities with a median LOS longer than 5 days compared with median LOS less than 4 days (AOR 0.88, p = 0.05). In other words, readmission was less likely in facilities with a practice of longer hospitalizations, adjusted for multiple patient characteristics, 
Table 1 Baseline patient characteristics for the Dialysis Outcomes and Practice Patterns Study (DOPPS) participants with at least one hospitalization $(n=8259)$

$\%$ or mean $\pm \mathrm{SD}^{*}$

Demographic characteristics

Age (years)

Black

$61.5 \pm 14.8$

Male

21.5

Median years on dialysis at start of study

56.0

Living status

Homeless

0.9

Living alone

0.2

Living with family or friend

15.3

Nursing home

76.5

Prisoner

5.9

Not known

2.0

Comorbid indicators

Cancer (other than skin)

10.7

Cerebrovascular disease

18.6

Congestive heart failure

40.5

Coronary heart disease

44.8

Diabetes

42.7

Dyspnea

30.3

Gastrointestinal bleeding

8.6

HIV/AIDS

1.3

Hypertension

80.9

Lung disease

Neurologic disease

Other cardiovascular disease

Peripheral vascular disease

Psychiatric disorder

25.0

Recurring cellulitis, gangrene

9.5

Patient measurements

Body mass index $\left(\mathrm{kg} / \mathrm{m}^{2}\right)$

$\mathrm{eKt} / \mathrm{V}_{\text {urea }}$ (adjusted for time of blood draw)

$24.3 \pm 5.4$

$1.2 \pm 0.3$

$5.7 \pm 1.9$

Phosphorus at enrollment

$3.6 \pm 0.6$

Albumin at enrollment

$31.0 \pm 5.3$

Type of vascular access

Fistula

37.2

24.4

Graft

11.0

Permanent catheter

9.1

*Unless specified to be median (years on dialysis); SD = standard deviation.

reason for hospitalization, index hospitalization LOS, and country.

For each country, the average median facility LOS and the average of the percentage of readmission in each facility were determined. Figure 2 shows an inverse correlation between the country average median facility
LOS and the average percentage of readmission (Pearson correlation, $r=-0.87$ ).

Table 4 shows the association between readmission and LOS, expressed as a continuous variable, for allDOPPS, US-DOPPS, Euro-DOPPS, and Japan-DOPPS. The AOR estimates the relative odds of readmission 
Table 2 Distribution of initial hospital admissions by diagnostic categories and percentage of readmissions within 30 days

\begin{tabular}{lccc}
\hline Reason for admission & $\begin{array}{c}\text { \% Initial admission } \\
(\mathrm{n}=21,434)\end{array}$ & $\begin{array}{c}\text { \% Readmitted } \\
(\mathrm{n}=5,624)\end{array}$ & $\begin{array}{c}\text { \% of All readmissions } \\
\text { due to the same reason }\end{array}$ \\
\hline Overall & 100 & 26.2 & 44.3 \\
Coronary heart disease & 8.4 & 27.2 & 40.2 \\
Other cardiac diseases & 9.3 & 27.7 & 38.4 \\
Vascular access & 22.6 & 26.8 & 56.7 \\
Infectious disease & 11.9 & 25.7 & 35.2 \\
Peripheral vascular disease & 6.3 & 30.8 & 50.4 \\
Gastrointestinal or hepatic disease & 9.2 & 24.7 & 40.9 \\
Pulmonary disease & 4.5 & 26.7 & 33.2 \\
Cerebrovascular or neurologic disease & 4.4 & 25.1 & 34.5 \\
Endocrine or metabolic disease & 3.6 & 26.2 & 36.9 \\
Musculoskeletal disease & 3.0 & 19.3 & 27.8 \\
Cancer & 1.8 & 33.1 & 66.4 \\
Psychiatric or mental illness & 1.2 & 32.0 & 18.8 \\
Health maintenance or routine care & 1.7 & 21.4 & 17.7 \\
Trauma or injury-related condition & 1.2 & 15.0 & 17.5 \\
Vision related & 0.7 & 12.2 & 27.8 \\
Missing reason & 10.0 & 25.6 & 54.5 \\
\hline
\end{tabular}

associated with one additional index hospital day and a 1-day increase in the median facility LOS. As in Fig. 1, each model was simultaneously adjusted for both hospital and median facility LOS. For all-DOPPS, the risk of readmission was approximately $0.5 \%$ higher for each additional index hospital day and approximately $2 \%$ to $3 \%$ lower for each additional median facility LOS. The associations were similar with and without adjustment for patient characteristics. The observed associations were stronger in the US compared with both Europe and Japan. In Europe and Japan, the associations were consistent but not statistically significant.

\section{DISCUSSION}

This study examined the association between hospital readmission and LOS. Early hospital readmission is usually an undesirable event and represents an important, if imperfect, quality indicator. The LOS of individual hospitalizations was considered an indicator of severity of illness and underlying patient status. In contrast, median dialysis facility LOS was used as an indicator of the facility practice pattern for LOS. Although median facility LOS was determined from individual hospital lengths of stay for the facility's patients, the two measures are statistically and conceptually distinct. In fact, readmissions were associated with these two LOS measures in very different ways.

For individual hospitalizations, the risk of readmission within 30 days of discharge was associated with longer individual hospital stays (Fig. 1A, Table 4). This finding was expected because longer hospital stays are generally associated with more serious illness, which, in turn, would increase the risk of subsequent hospitalization.

Table 3 Distribution of median facility hospital length of stay (LOS), overall, and by Dialysis Outcomes and Practice Patterns Study (DOPPS) location

\begin{tabular}{|c|c|c|c|c|}
\hline & All DOPPS & US-DOPPS & Euro-DOPPS & Japan-DOPPS \\
\hline Median of median facility LOS (days) & 5.0 & 4.0 & 5.0 & 12.5 \\
\hline Average of median facility LOS (days) & 6.9 & 4.3 & 6.6 & 13.6 \\
\hline \multicolumn{5}{|l|}{ Median facility LOS (\% of facilities) } \\
\hline$<4$ days & 19.0 & 23.0 & 22.0 & 3.0 \\
\hline 4-5 days & 38.0 & 62.0 & 26.0 & 2.0 \\
\hline$>5$ days & 43.0 & 15.0 & 52.0 & 95.0 \\
\hline
\end{tabular}



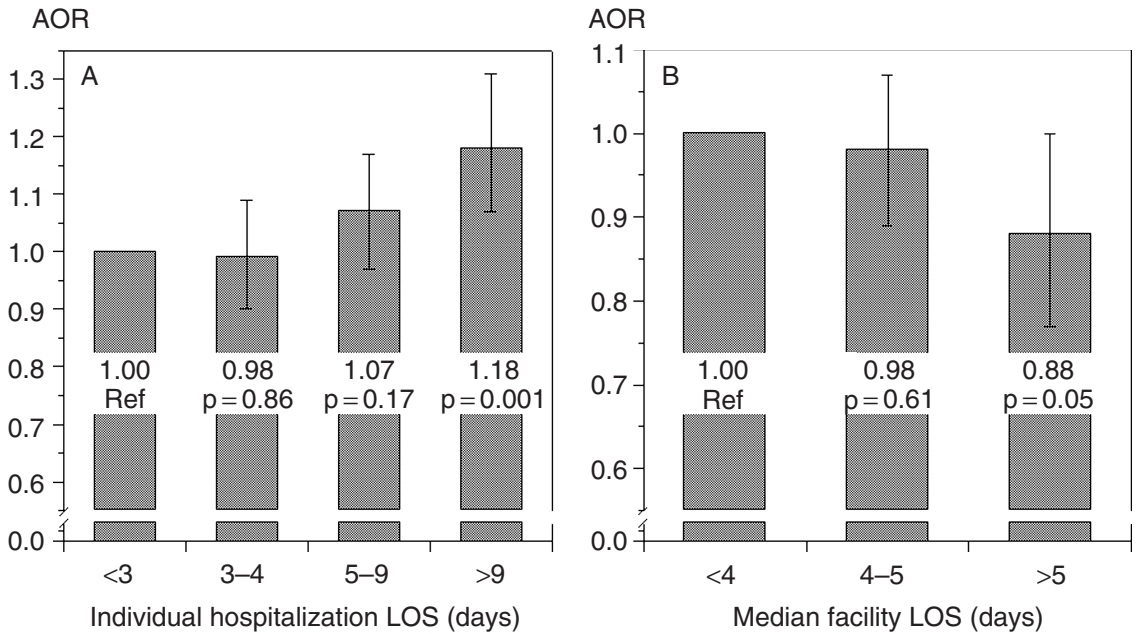

Figure 1 The odds of hospital readmission (expressed as the adjusted odds ratio, AOR) was directly associated with hospital length of stay (LOS, A) and inversely associated with median facility LOS (B).

By contrast, after adjustment for multiple patient characteristics and the index hospitalization LOS, readmission risk was inversely associated with the median facility LOS (Fig. 1B, Table 4). As noted, median facility LOS is an integrated measure of local hospitalization practice, as determined by the practitioners, hospitals, and treatment customs associated with each dialysis facility. These

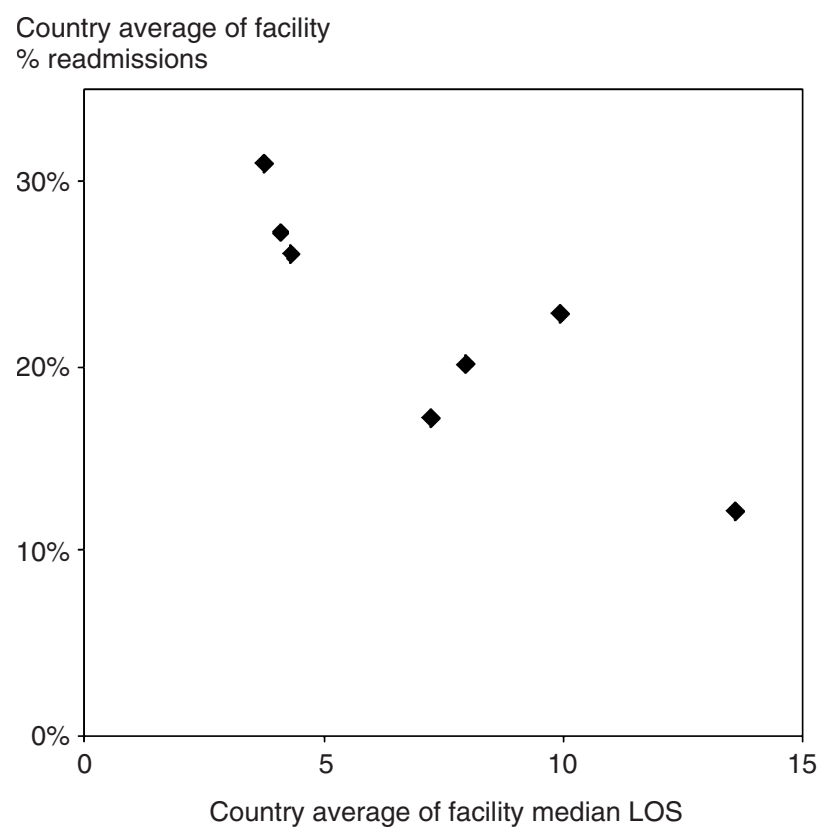

Figure 2 Association between average dialysis facility readmission rate and average median length of stay (LOS) for each country in the DOPPS (Pearson correlation, $r=-0.87$ ). results are consistent with the hypothesis that the risk of hospital readmission is higher for patients who receive dialysis treatment in facilities where a shorter hospital LOS is the generally adopted practice. If variations in facility median LOS were primarily the result of facility differences in patient characteristics rather than in practice, then one might expect no facility LOS effect in models that were adjusted for multiple patient characteristics.

Early readmission has been interpreted as an indicator of the quality of hospital and posthospital care. ${ }^{11}$ It is recognized that some early readmissions are expected for such situations as staged surgical interventions, cancer therapy, and procedures that were appropriately scheduled during a prior hospitalization. ${ }^{21}$ In contrast, unplanned early readmission suggests the possibility of premature discharge or suboptimal care during the hospitalization or following discharge. ${ }^{10,11,22-24}$ Potential factors that may increase the risk of early readmission include lack of diagnostic resources, incorrect treatment selection, inadequate nursing care, nosocomial infections, and a focus on a single problem in patients with multiple comorbid conditions. Readmission risk is also related to patient characteristics and the primary disease process.

In this analysis it was not possible to differentiate planned from unplanned admissions. ${ }^{25}$ We observed, however, a higher risk of readmission for hospitalizations associated with certain disease categories such as cancer, peripheral vascular diseases, and cardiac diseases (Table 2). A fraction of early readmissions among these patients likely were planned. A more in-depth analysis of these hospitalizations is necessary to identify the factors that 
Table 4 Associations between readmission and hospital length of stay (LOS), based on individual hospitalization LOS and facility LOS

\begin{tabular}{|c|c|c|c|c|}
\hline & \multicolumn{2}{|c|}{$\begin{array}{c}\text { Odds ratio (95\% confidence interval) } \\
\text { per 1-day increase in individual } \\
\text { hospitalization LOS }\end{array}$} & \multicolumn{2}{|c|}{$\begin{array}{c}\text { Odds ratio ( } 95 \% \text { confidence interval) } \\
\text { per 1-day increase in median } \\
\text { facility LOS }\end{array}$} \\
\hline & Unadjusted & Adjusted* & Unadjusted & Adjusted* \\
\hline All-DOPPS & $\begin{array}{c}1.006(1.002-1.009) \\
p<0.001\end{array}$ & $\begin{array}{c}1.005(1.001-1.008) \\
p=0.007\end{array}$ & $\begin{array}{c}0.979(0.959-1.000) \\
p=0.050\end{array}$ & $\begin{array}{c}0.974(0.954-0.995) \\
p=0.016\end{array}$ \\
\hline US-DOPPS & $\begin{array}{c}1.008(1.004-1.012) \\
p<0.001\end{array}$ & $\begin{array}{c}1.007(1.003-1.012) \\
p=0.001\end{array}$ & $\begin{array}{c}0.959(0.924-0.996) \\
p=0.028\end{array}$ & $\begin{array}{c}0.954(0.919-0.991) \\
p=0.015\end{array}$ \\
\hline Euro-DOPPS & $\begin{array}{c}1.004(0.999-1.010) \\
p=0.097\end{array}$ & $\begin{array}{c}1.003(0.998-1.009) \\
p=0.276\end{array}$ & $\begin{array}{c}1.010(0.972-1.049) \\
p=0.606\end{array}$ & $\begin{array}{c}0.996(0.958-1.036) \\
p=0.852\end{array}$ \\
\hline Japan-DOPPS & $\begin{array}{c}1.005(0.989-1.021) \\
p=0.571\end{array}$ & $\begin{array}{c}0.999(0.989-1.009) \\
p=0.885\end{array}$ & $\begin{array}{c}0.980(0.945-1.016) \\
p=0.270\end{array}$ & $\begin{array}{c}0.994(0.962-1.026) \\
p=0.693\end{array}$ \\
\hline
\end{tabular}

*Adjusted for all variables listed in Table 1 and for country. All models include both patient LOS and facility median LOS.

contribute to unplanned readmissions. This analysis, controlled for multiple factors that could plausibly influence the risk of readmission, suggests that the inverse association between shorter median facility LOS and higher readmission risk stems partly from a systematic tendency toward premature discharge of patients treated at some dialysis facilities, particularly in the United States.

The association between shorter median facility LOS and higher odds of readmission was strongest in the US-DOPPS (Table 4). This finding may be related to greater pressure to shorten LOS for dialysis patients in the United States compared with Europe and, particularly, Japan (Table 3, Fig. 2). ${ }^{4,26}$ The associations were weaker and nonsignificant in Euro-DOPPS and JapanDOPPS, likely in part because of longer facility LOS (Fig. 2) and/or smaller sample size. It is possible that the trend toward shorter LOS for dialysis patients in the United States is driven by the Medicare prospective payment system, which provides a financial incentive for shorter hospital stays. ${ }^{27}$ The incentive to shorten LOS is apparently weaker in other countries, particularly in Japan. $^{26,28}$ In fact, the longer hospital LOS in Japan seems to be a general phenomenon rather than a finding specific for hemodialysis patients. It is possible that the hospital LOS practices in Europe and Japan exceed the threshold at which a measurable impact on readmission risk can be detected.

These data do not permit an assessment of the cost tradeoff between shorter LOS and higher readmission risk. Others have shown a diminishing impact of incremental hospital days on total hospitalization costs. ${ }^{29}$ Nevertheless, hospitalization costs per dialysis patient have increased over time even as LOS has declined in the United States. ${ }^{4}$ The increased risk of early readmission in dialysis facilities with lower median LOS raises important questions about the quality of in-hospital care and discharge planning for dialysis patients.

In conclusion, our findings indicate that patients treated in dialysis facilities with lower median LOS present higher odds of readmission when taking into account disease severity and reason for admission. The association was stronger in the United States, where there is a great emphasis to reduce LOS. The increase in the odds of readmission in facilities with shorter median LOS suggests incomplete resolution of the problems of hospitalized hemodialysis patients, particularly in the United States. Hospitalization practice may be better tailored to patient needs in Euro-DOPPS and Japan-DOPPS than in US-DOPPS. These findings, based on observational data from a sample of dialysis patients and facilities, should be confirmed using other sources of hospital data. Additional research is needed to understand the quality and cost implications of hospital LOS practices.

\section{ACKNOWLEDGMENTS}

The authors express appreciation to members of the Worldwide DOPPS Committee for contributions during the design and implementation of the DOPPS. For a full listing of committee members, please see Young et al. ${ }^{19}$ The DOPPS is supported by research grants from Amgen, Inc., and Kirin Brewery, Ltd., without restrictions on publications. A.A.L. was supported by a grant (BEX2018/00-4) from the Fundação Coordenação de Aperfeiçoamento de Pessoal de Nível Superior (CAPES Foundation), Ministry of Education of Brazil. 


\section{REFERENCES}

1 Woods JD, Port FK. The impact of vascular access for haemodialysis on patient morbidity and mortality. Nephrol Dial Transplant. 1997; 12(4):657-659.

2 Dhingra RK, Young EW, Hulbert-Shearon TE, Leavey SF, Port FK. Type of vascular access and mortality in U.S. hemodialysis patients. Kidney Int. 2001; 60(4): 1443-1451.

3 Port FK. Morbidity and mortality in dialysis patients. Kidney Int. 1994; 46(6):1728-1737.

4 United States Renal Data System. Hospitalization in ESRD. Am J Kidney Dis. 1999; 34(2 Suppl 1): S114-S123.

5 United States Renal Data System. USRDS 2001 Annual Data Report [Internet]. Bethesda, MD: National Institutes of Health, National Institute of Diabetes and Digestive and Kidney Diseases, 2001. Available from: http:// www.usrds.org/adr_2001.htm

6 Schwartz WB, Mendelson DN. Hospital cost containment in the 1980s: Hard lessons learned and prospects for the 1990s. N Engl J Med. 1991; 324(15):1037-1042.

7 Durbin CG Jr, Kopel RF. A case-control study of patients readmitted to the intensive care unit. Crit Care Med. 1993; 21(10):1547-1553.

8 Hofer TP, Hayward RA. Can early re-admission rates accurately detect poor-quality hospitals? Med Care. 1995; 33(3):234-245.

9 Cline CM, Broms K, Willenheimer RB, Israelsson BA, Erhardt LR. Hospitalization and health care costs due to congestive heart failure in the elderly. Am J Geriatr Cardiol. 1996; 5(4):10-14.

10 Polanczyk CA, Newton C, Dec GW, Di Salvo TG. Quality of care and hospital readmission in congestive heart failure: An explicit review process. J Card Fail. 2001; 7(4):289-298.

11 Ashton CM, Del Junco DJ, Souchek J, Wray NP, Mansyur $\mathrm{CL}$. The association between the quality of inpatient care and early readmission: A meta-analysis of the evidence. Med Care. 1997; 35(10):1044-1059.

12 Nassar GM, Ayus JC. Infectious complications of the hemodialysis access. Kidney Int. 2001; 60(1):1-13.

13 Foley RN, Parfrey PS, Harnett JD, Kent GM, Murray DC, Barre PE. Hypoalbuminemia, cardiac morbidity, and mortality in end-stage renal disease. J Am Soc Nephrol. 1996; 7(5):728-736.

14 Ganesh SK, Stack AG, Levin NW, Hulbert-Shearon T, Port FK. Association of elevated serum $\mathrm{PO}_{4}, \mathrm{Ca} \times \mathrm{PO}_{4}$ product, and parathyroid hormone with cardiac mortality risk in chronic hemodialysis patients. J Am Soc Nephrol. 2001; 12(10):2131-2138.

15 Harnett JD, Foley RN, Kent GM, Barre PE, Murray D, Parfrey PS. Congestive heart failure in dialysis patients: Prevalence, incidence, prognosis and risk factors. Kidney Int. 1995; 47(3):884-890.
16 United States Renal Data System. Comorbid conditions and correlations with mortality risk among 3,399 incident hemodialysis patients. Am J Kidney Dis. 1992; 20(5 Suppl 2):32-38.

17 Jaar BG, Hermann JA, Furth SL, Briggs W, Powe NR. Septicemia in diabetic hemodialysis patients: Comparison of incidence, risk factors, and mortality with nondiabetic hemodialysis patients. Am J Kidney Dis. 2000; 35(2): 282-292.

18 Ward MM. Cardiovascular and cerebrovascular morbidity and mortality among women with end-stage renal disease attributable to lupus nephritis. Am J Kidney Dis. 2000; 36(3):516-525.

19 Young EW, Goodkin DA, Mapes DL et al. The Dialysis Outcomes and Practice Patterns Study (DOPPS): An international hemodialysis study. Kidney Int. 2000; 57(Suppl. 74):74-81.

20 Daugirdas JT, Depner TA, Gotch FA, Greene T, Keshaviah P, Levin NW, Schulman G. Comparison of methods to predict equilibrated Kt/V in the HEMO Pilot Study. Kidney Int. 1997; 52(5):1395-1405.

21 Ashton CM, Kuykendall DH, Johnson ML, Wray NP, Wu L. The association between the quality of inpatient care and early readmission. Ann Intern Med. 1995; 122(6): 415-421.

22 Weinberger M, Smith DM, Katz BP, Moore PS. The cost-effectiveness of intensive postdischarge care: A randomized trial. Med Care. 1988; 26(11):1092-1102.

23 Martens KH, Mellor SD. A study of the relationship between home care services and hospital readmission of patients with congestive heart failure. Home Health Nurse. 1997; 15(2):123-129.

24 Handy JR Jr,, Child AI, Grunkemeier GL, Fowler P, Asaph JW, Douville EC, Tsen AC, Ott GY. Hospital readmission after pulmonary resection: Prevalence, patterns, and predisposing characteristics. Ann Thorac Surg. 2001; 72(6):1855-1859.

25 Marcantonio ER, McKean S, Goldfinger M, Kleefield S, Yurkofsky M, Brennan TA. Factors associated with unplanned hospital readmission among patients 65 years of age and older in a Medicare managed care plan. Am J Med. 1999; 107(1):13-17.

26 Muramatsu N, Liang J. Hospital length of stay in the United States and Japan: A case study of myocardial infarction patients. Int J Health Serv. 1999; 29(1): 189-209.

27 Fetter RB, Shin Y, Freeman JL, Averill RF, Thompson JD. Case mix definition by diagnosis-related groups. Med Care. 1980; 18(2 Suppl): iii, 1-53.

28 Yamaoka K, Kobayashi Y, Yano E. Length of in-patient stay in teaching hospitals in Japan. Med Educ. 1993; 27(3):280-285.

29 Taheri PA, Butz DA, Greenfield LJ. Length of stay has minimal impact on the cost of hospital admission. J Am Coll Surg 2000; 191(2):123-130. 